\title{
Review
}

\section{HIV-1 Nuclear Import: Matrix Protein Is Back on Center Stage, This Time Together with Vpr}

\author{
Michael I. Bukrinsky ${ }^{1}$ and Omar K. Haffar ${ }^{2}$ \\ ${ }^{1}$ The Picower Institute for Medical Research, Manhasset, New York, \\ U.S.A. \\ ${ }^{2}$ Cytokine Networks Inc., Seattle, Washington, U.S.A.
}

\section{Introduction}

The ability of HIV-1 to transport its pre-integration complex (PIC) into the nucleus of an infected cell during interphase is a unique feature that sets this virus, and the whole group of Lentiviruses to which it belongs, apart from other retroviruses that have to rely on the dissolution of the nuclear envelope during mitosis for delivery of their genome into the nucleus $(1,2)$. Following initial reports demonstrating the central role of this process in the ability of HIV-l to replicate in nondividing cells, such as macrophages (3-5), the mechanisms of HIV-1 nuclear import have become the subject of intensive research. Early results (6) indicated that the process of nuclear import of HIV-1 genome is energy dependent, thus implying an active transport mechanism and suggesting that the virus is exploiting the cellular nuclear import machinery.

This machinery transports into the nucleus those cellular proteins that carry characteristic nuclear localization signals (NLSs). The most common type of NLS is a short stretch of basic amino acids that introduce an overall net positive charge crucial for nuclear targeting properties of these sequences (reviewed in ref. 7). Import of basic-type NLS-containing proteins across the nuclear pore complex is mediated by karyopherin $\alpha / \beta$ heterodimers (also termed NLS receptor/importin) which bind NLS-containing proteins in the cytosol and target them to the nucleus (8-11). Karyopherin $\alpha$ binds the NLS, whereas karyopherin $\beta$ enhances the affinity of $\alpha$

Address correspondence and reprint requests to: Dr. Michael I. Bukrinsky, The Picower Institute for Medical Research, 350 Community Drive, Manhasset, NY 11030 , U.S.A. Phone: (516) 365-4200; Fax: (516) 365-0286;

E-mail: mbukrinsky@picower.edu for the NLS and mediates docking of the import complex to nucleoporins (a collective term for nuclear pore complex proteins) that contain XxFG peptide repeats (reviewed in ref. 12). A small GTP-binding protein, $\operatorname{Ran} / \mathrm{TC} 4(13,14)$, is probably the major regulator of the directionality of nuclear transport (15). The direct binding of RanGTP to karyopherin $\beta$ terminates the translocation by disassembling the import complex (16-18). In addition to karyopherins and Ran, several other soluble proteins are involved in nuclear import, although their mechanism of action is less defined. The nuclear import factor pl0 (also termed NTF2) $(19,20)$ appears to coordinate the activity of Ran by binding Ran-GDP into a complex with nucleoporin-docked karyopherins (21). Heat-shock protein 70 (Hsp70, Hsc70), as well as some as yet uncharacterized cytoplasmic factors, may act to facilitate the interaction between the NLS and karyopherin $\alpha$ $(22,23)$. The ectopic expression of human Hsp70 in mouse cells complemented the defective import of a mutant SV40 large T antigen (24), and the depletion of Hsp70 from cytosolic extracts prevented import $(25,26)$.

A search for NLS-containing HIV-1 proteins that could target the viral PIC for interaction with the cellular import machinery resulted in quick success. A basic-type NLS was identified in the N-terminal region of the HIV-1 matrix protein (MA) (27) and was found to regulate viral nuclear import and infection of nondividing cells $(4,27)$. However, the role of MA in HIV-1 nuclear import was disputed in later publications $(28,29)$. Another HIV-1 protein with a basic-type NLS is integrase (30), although its role in nuclear import is also controversial $(30,31)$. Genetic analysis also implicated viral protein $\mathrm{R}(\mathrm{Vpr})$ in 
the process of nuclear import of the HIV-1 PIC (3-5). But this protein does not have a classical NLS, and its role in nuclear import remained unclear until recently (32).

In this article we critically review previously published reports on the role of HIV-1 proteins in the process of nuclear import of the viral PIC in light of the most recent information from our and other laboratories. The emerging model depicts MA and Vpr as the main regulators of HIV-1 nuclear import and facilitates development of novel inhibitors of this process.

\section{HIV-1 Proteins that Regulate Nuclear Import of the Viral PIC}

The MA protein was the first to be identified as a participant from the viral side in the process of HIV-1 nuclear import (27). Its role turned out to be also the most controversial. The work by Bukrinsky et al. (27) and by Nadler et al. (22) demonstrated that a basic region in the MA protein encompassing amino acids 25-33, $\mathrm{G}^{25} \mathrm{KKKYKLKH}$, functions as an NLS when conjugated to BSA. The role of this region in HIV-1 nuclear import was supported by genetic experiments that found a correlation between the inability of HIV-1 variants carrying inactivating mutations in this NLS to import their PIC into the nucleus and poor replication of such viruses in nondividing cells $(4,5,27)$. However, these findings were challenged by two other groups $(28,29)$ who reported only a minor defect in replication of MA NLS mutant viruses and suggested that it resulted from abnormal processing of the Gag polyprotein, rather than from a nuclear import defect. One of the arguments used in those studies to dispute the role of the MA NLS in HIV-1 nuclear import was that NLS mutations caused an almost similar attenuation of viral replication in nondividing (e.g., macrophages) and dividing (e.g., activated $\mathrm{T}$ lymphocytes) cells $(28,29)$. This argument relies on the hypothesis that in dividing cells, such as activated $\mathrm{T}$ lymphocytes, entry of the virus into the nucleus occurs during mitosis and thus does not require active nuclear importation; this hypothesis is based primarily on the ability of importdeficient mutants to replicate in $\mathrm{CD}^{+} \mathrm{T}$ cell lines $(3,6,27)$. However, published evidence indicates that active nuclear import mechanism can be functional in HIV-1 infection of immortalized T cells, at least under certain conditions. For in- stance, while HIV-1 does not replicate in quiescent $\left(G_{0}\right)$ T lymphocytes $(33,34)$, it can productively infect $T$ cells arrested in either $G_{1}-S(35)$ or $G_{2}(5)$ phases of the cell cycle, suggesting that cell activation, but not cell division, is necessary for virus replication in $\mathrm{T}$ cells. Most importantly, recent results (36) obtained using an inhibitor of HIV-1 nuclear translocation indicate that the ability of the PIC to engage in active nuclear import is critical for HIV-1 replication in primary activated $T$ lymphocytes. It thus appears that while active nuclear import may be less critical for HIV-1 infection of rapidly dividing cells, it still provides a significant advantage for viral replication, especially in cells with a relatively long interphase. Therefore, reported defects in replication of the MA NLS-defective HIV-1 in activated $\mathrm{T}$ lymphocytes $(28,29)$ might still be caused by inefficient nuclear import.

Some of the controversy surrounding the role of MA in HIV-1 nuclear import may also be explained by an inherent weakness of MA as a karyophile. Compared with the NLS of SV40 large $\mathrm{T}$ antigen, the N-terminal MA NLS is a weak one, requiring the presence of multiple peptides per BSA molecule to achieve partial nuclear localization $(22,27)$. This may be the reason that one report (29) denied any karyophilic potential of the MA protein. This, of course, is a gross exaggeration, given a well-documented ability of MA to bind to karyopherin $\alpha(32,37,38)$ and to direct nuclear import of the fusion protein (39), but it illustrates the weakness of MA as a karyophile. How then can it target to the nucleus a large macromolecular complex such as the HIV-1 PIC? To some extent, the weakness of the MA NLS is compensated by the presence of multiple $(\approx 1000)$ copies of MA in the HIV-1 PIC (40). Presence of multiple NLSs has been shown to improve substantially nuclear import, especially of large molecules (41). In addition, other proteins within the PIC [e.g., integrase (39)] may contribute their NLSs to the process of HIV nuclear import. However, multiplicity of NLSs on the HIV-1 PIC is not sufficient to make it a strong karyophile without involvement of another viral protein, Vpr.

The role of Vpr in HIV-1 nuclear import was suggested by experiments that demonstrated that in the presence of a functional vpr gene, the effect of inactivating mutations in the MA NLS on nuclear import was greatly diminished $(3,5)$. Vpr rescued replication of an MA NLS mutant in macrophages by providing sufficient, although 
reduced by about $50-80 \%$ compared with the wild-type virus, nuclear translocation of the viral DNA $(3,39,42)$. In addition, Vpr was shown to target a fusion protein to the nuclear envelope (43), which is consistent with its role in nuclear import. However, the mechanism of Vpr's activity remained unclear until recently. The Vpr protein does not have a canonical basic-type NLS, and the nuclear targeting determinant appears to reside in the $\alpha$-helical region of the protein $(44,45)$. This region is also involved in mediating Vpr interactions with cellular protein(s) $(46,47)$. Mutations in the $\alpha$-helix domain of $\mathrm{Vpr}$ that abolished protein-protein interactions also affected nuclear localization of $\mathrm{Vpr}(45,48)$. It appears, therefore, that karyophilic properties of Vpr are mediated by a cellular Vpr-interacting protein.

This protein was recently identified as karyopherin $\alpha(32,43)$. An earlier study by Gallay et al. (38), based on indirect evidence from experiments using dominant-negative mutant of karyopherin $\alpha$, concluded that Vpr controls nuclear import through a karyopherin $\alpha$-independent mechanism. This conclusion was consistent with resistance of Vpr-regulated HIV-1 nuclear import to competition by NLS peptides (38). Some of these earlier results can be explained by an unusual mode of interaction between Vpr and karyopherin $\alpha$. Whereas binding of MA and other karyophiles to karyopherin $\alpha$ is mediated by the basic-type NLS, binding of Vpr to $\alpha$ does not involve an NLS (32). Therefore, the binding site of Vpr on karyopherin $\alpha$ does not appear to overlap with the NLS binding sites; in fact, karyopherin $\alpha, \mathrm{Vpr}$, and MA can assemble into a trimer (32). As a result of Vpr binding to karyopherin $\alpha$, the affinity of interaction between the NLS and $\alpha$ was increased approximately 10-fold. This mechanism may be instrumental in increasing the karyophilic potential of the HIV-1 preintegration complex.

Significant progress in the studies of HIV-l nuclear import was achieved with the introduction of an in vitro system that truly reproduced this process (32). Using this system, Popov et al. (32) demonstrated that inactivation of Vpr completely knocked out import even in the presence of functional MA NLS. This result appears to be inconsistent with in vivo studies that reported Vpr-independent infection of nondividing cells, such as neurons $(42,49)$, or growth-arrested HeLa and T cells $(3,5,39)$. An explanation for this paradox is provided by the finding that cytoplas- mic lysates of HeLa cells can rescue import of a Vpr-defective virus in vitro (32), suggesting existence of cellular proteins with a Vpr-like function. The nature of these proteins remains to be identified, as does their mechanism of action, which may or may not be similar to the Vpr's activity.

Another interesting conclusion from the experiments employing in vitro import assay was that in the presence of Vpr, mutation in the MA NLS had only a small effect on nuclear import of the HIV-1 PIC (32). In addition, Vpr enhanced nuclear import of an artificial karyophile (BSA conjugated with the NLS peptide), suggesting that Vpr can stimulate karyophilic activity of any basic-type NLS. This conclusion has important implications for the model of HIV-1 nuclear import. First of all, it explains earlier results $(28,29)$ that showed only a small effect of MA NLS mutation on HIV-1 replication in nondividing macrophages. Secondly, it suggests that some weak NLSs within the HIV-1 PIC, the effect of which could be missed in classical import assays, may function in the presence of Vpr. One such NLS was recently identified in the C-terminal part of the MA protein $\left({ }^{109} \mathrm{KSKKK}\right)(22)$. When the $\mathrm{N}$ terminal MA NLS was mutated, this NLS could target the HIV-1 PIC into the nucleus in vitro, but only in the presence of Vpr (Popov et al., unpublished results). Mutation of both MA NLSs resulted in complete loss of nuclear import, regardless of the Vpr presence. Such virus was also unable to replicate in macrophages (S. Popov and $\mathrm{M}$. Bukrinsky, unpublished results). Interestingly, both MA NLSs are located in close proximity on a crystal structure of the protein (50), suggesting that they may function as a bi-partite NLS.

These results underscore the central role of the MA protein in HIV-1 nuclear import and provide an alternative explanation for some of previously reported results. Indeed, all studies addressing the function of MA in HIV-l infection and nuclear import ignored the second (C-terminal) MA NLS, supposing that inactivation of the $\mathrm{N}$-terminal NLS abolishes the karyophilic potential of MA. For instance, this logic was used in a recent report (39) implicating HIV-1 integrase (IN) in nuclear import of the PIC. Although the role of the IN NLS in HIV-1 nuclear import can not be ruled out completely, it is inconsistent with the lack of import of the HIV-1 PIC carrying mutations in both NLSs (S. Popov and M. Bukrinsky, unpublished results). 


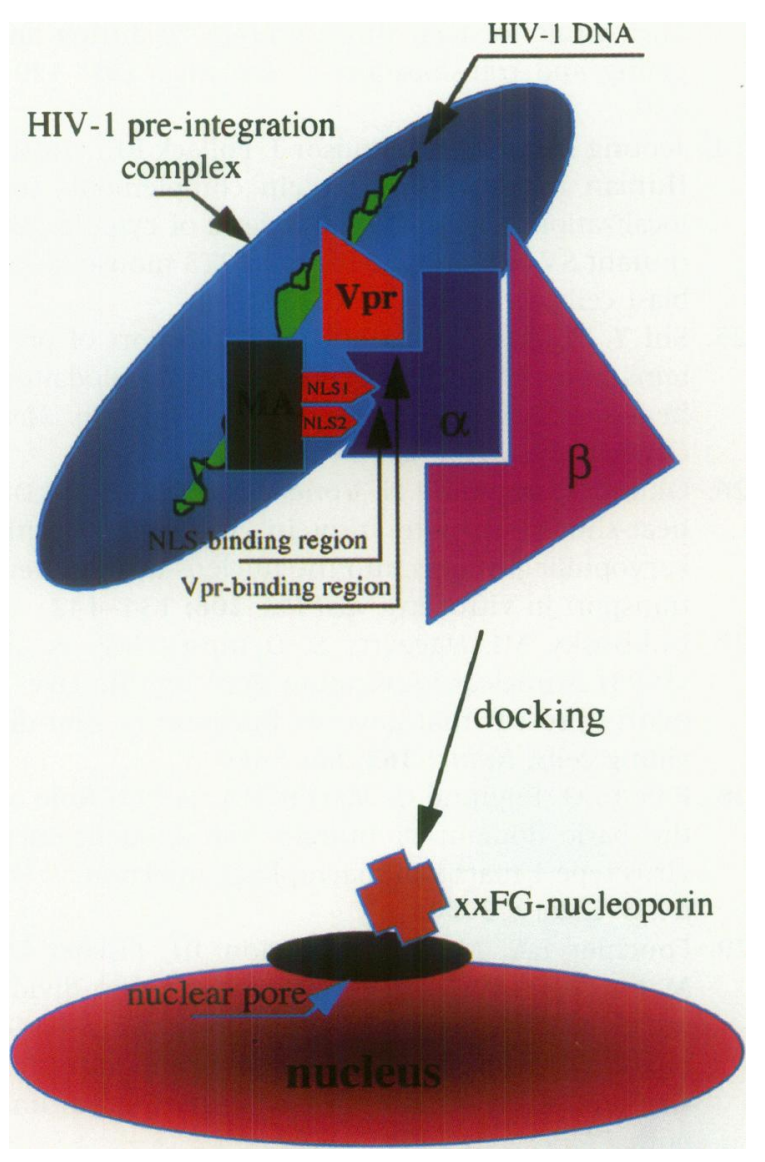

FIG. 1. A model of HIV-1 nuclear import. Nuclear import of the HIV-1 PIC is driven by the cellular proteins karyopherin $\alpha$ and karyopherin $\beta$. Two MA NLSs (NLS1 and NLS2) provide the connection between the PIC and karyopherin $\alpha$. NLSI (amino acids 26-32) is a stronger NLS than NLS2 (amino acids 109-113), but they may cooperate to form a bipartite NLS, thus interacting with the same region on karyopherin $\alpha$. Alternatively, they may bind to adjacent NLS-binding sites on karyopherin $\alpha$. The role of $\mathrm{Vpr}$ is to strengthen interaction between the MA NLSs and karyopherin $\alpha$. This is likely achieved by a conformational change in the karyopherin- $\alpha$ molecule upon binding of $\mathrm{Vpr}$. This binding involves a site different from the NLS-binding site on $\alpha$. The import complex is then targeted to the nuclear pore via interaction between karyopherin $\beta$ and xxFGrepeat-containing nucleoporins. Other details are in the text.

\section{Conclusions}

It appears, therefore, that nuclear import of the HIV-l PIC is controlled by two viral proteins, Vpr and MA (Fig. 1). While MA contributes its two NLSs which connect the PIC to karyopherin $\alpha$ and cellular import machinery, $\mathrm{Vpr}$ functions as an enhancer of the MA-karyopherin $\alpha$ interaction. In addition, Vpr was found to bind yeast nucleoporins (43) and was suggested on these grounds to function as a karyopherin $\beta$ analog. Although this hypothesis is consistent with the role of Vpr in docking of the HIV-1 PIC to the nuclear envelope (51), it is hard to imagine that a small protein, such as Vpr (with a molecular mass of only $14 \mathrm{kDa}$ ), can perform the function of a $97-\mathrm{kDa}$ karyopherin $\beta$. This hypothesis is also inconsistent with our results showing that karyopherin $\alpha$, karyopherin $\beta, \mathrm{Vpr}$, and MA assemble into a tetrameric complex (S. Popov and M. Bukrinsky, unpublished results). We therefore favor the notion that the effects of Vpr are mediated through the karyopherin $\alpha / \beta$ pathway. Inactivation of either partner in the HIV-1 nuclear import (i.e., MA, Vpr, or karyopherin $\alpha / \beta$ ) results in the loss of import function and virus inability to establish efficient infection in primary cells. These proteins, therefore, present an attractive target for development of novel anti-HIV therapeutics.

\section{Acknowledgments}

We thank members of the Bukrinsky lab for helpful discussions. This work was supported by NIH grants AI 33776 and AI 40386 and by funds from The Picower Institute for Medical Research.

\section{References}

1. Lewis PF, Emerman M. (1994) Passage through mitosis is required for oncoretroviruses but not for the human immunodeficiency virus. J. Virol. 68: 510-516.

2. Roe T, Reynolds TC, Yu G, Brown PO. (1993) Integration of murine leukemia virus DNA depends on mitosis. EMBO J. 12: 2099-2108.

3. Heinzinger NK, Bukrinsky MI, Haggerty SA, et al. (1994) The Vpr protein of human immunodeficiency virus type 1 influences nuclear localization of viral nucleic acids in nondividing host cells. Proc. Natl. Acad. Sci. USA 91: 7311-7315.

4. von Schwedler U, Kornbluth RS, Trono D. (1994) The nuclear localization signal of the matrix protein of human immunodeficiency virus type 1 allows the establishment of infection in macrophages and quiescent T lymphocytes. Proc. Natl. Acad. Sci. USA 91: 6992-6996.

5. Emerman M, Bukrinsky M, Stevenson M. (1994) HIV-1 infection of non-dividing cells. Nature 369: 107-108.

6. Bukrinsky MI, Sharova N, Dempsey MP, et al. (1992) Active nuclear import of human immunodeficiency virus type 1 preintegration complexes. Proc. Natl. Acad. Sci. USA 89: 6580-6584. 
7. Dingwall C, Laskey RA. (1991) Nuclear targeting sequences-a consensus? Trends Biochem. Sci. 16: 478-481.

8. Adam SA, Gerace L. (1991) Cytosolic proteins that specifically bind nuclear location signals are receptors for nuclear import. Cell 66: 837-847.

9. Gorlich D, Kostka S, Kraft R, et al. (1995) Two different subunits of importin cooperate to recognize nuclear localization signals and bind them to the nuclear envelope. Curr. Biol. 5: 383-392.

10. Imamoto N, Shimamoto T, Kose S, et al. (1995) The nuclear pore-targeting complex binds to nuclear pores after association with a karyophile. FEBS Lett. 368: 415-419.

11. Enenkel C, Blobel G, Rexach M. (1995) Identification of a yeast karyopherin heterodimer that targets import substrate to mammalian nuclear pore complexes. J. Biol. Chem. 270: 16499-16502.

12. Gorlich D. (1997) Nuclear protein import. Curr. Opin. Cell Biol. 9: 412-419.

13. Moore MS, Blobel G. (1993) The GTP-binding protein Ran/TC4 is required for protein import into the nucleus. Nature 365: 661-663.

14. Melchior F, Paschal B, Evans J, Gerace L. (1993) Inhibition of nuclear protein import by nonhydrolyzable analogues of GTP and identification of the small GTPase Ran/TC4 as an essential transport factor. J. Cell Biol. 123: 1649-1659.

15. Gorlich D, Kraft R, Kostka S, et al. (1996) Importin provides a link between nuclear protein import and U snRNA export. Cell 87: 21-32.

16. Gorlich D, Pante N, Kutay U, Aebi U, Bischoff FR. (1996) Identification of different roles for RanGDP and RanGTP in nuclear protein import. EMBO J. 15: $5584-5594$.

17. Rexach M, Blobel G. (1995) Protein import into nuclei: Association and dissociation reactions involving transport substrate, transport factors, and nucleoporins. Cell 83: 683-692.

18. Chi NC, Adam EJ, Visser GD, Adam SA. (1996) RanBP1 stabilizes the interaction of Ran with p97 nuclear protein import. J. Cell Biol. 135: 559-569.

19. Moore MS, Blobel G. (1994) Purification of a Raninteracting protein that is required for protein import into the nucleus. Proc. Natl. Acad. Sci. USA 91: 10212-10216.

20. Paschal BM, Gerace L. (1995) Identification of NTF2, a cytosolic factor for nuclear import that interacts with nuclear pore complex protein p62. J. Cell Biol. 129: 925-937.

21. Nehrbass U, Blobel G. (1996) Role of the nuclear transport factor plo in nuclear import. Science 272: 120-122.

22. Nadler SG, Tritschler D, Haffar OK, Blake J, Bruce AG, Cleaveland JS. (1997) Differential expression and sequence-specific interaction of karyopherin alpha with nuclear localization sequences. J. Biol. Chem. 272: 4310-4315.

23. Shulga N, Roberts P, Gu Z, et al. (1996) In vivo nuclear transport kinetics in Saccharomyces cerevi- siae: A role for heat shock protein 70 during targeting and translocation. J. Cell Biol. 135: 329339.

24. Jeoung DI, Chen S, Windsor J, Pollack RE. (1991) Human major HSP70 protein complements the localization and functional defects of cytoplasmic mutant SV40 T antigen in Swiss 3T3 mouse fibroblast cells. Genes Dev. 5: 2235-2244.

25. Shi Y, Thomas JO. (1992) The transport of proteins into the nucleus requires the 70-kilodalton heat shock protein or its cytosolic cognate. Mol. Cell Biol. 12: 2186-2192.

26. Okuno Y, Imamoto N, Yoneda Y. (1993) 70-kDa heat-shock cognate protein colocalizes with karyophilic proteins into the nucleus during their transport in vitro. Exp. Cell Res. 206: 134-142.

27. Bukrinsky MI, Haggerty S, Dempsey MP, et al. (1993) A nuclear localization signal within HIV-1 matrix protein that governs infection of non-dividing cells. Nature 365: 666-669.

28. Freed EO, Englund G, Martin MA. (1995) Role of the basic domain of human immunodeficiency virus type 1 matrix in macrophage infection. $J$. Virol. 69: 3949-3954.

29. Fouchier RA, Meyer BE, Simon JH, Fischer U, Malim MH. (1997) HIV-1 infection of non-dividing cells: Evidence that the amino-terminal basic region of the viral matrix protein is important for Gag processing but not for post-entry nuclear import. $E M B O$ J. 16: 4531-4539.

30. Madurai S, Moodley D, Coovadia HM, Gopaul W, Smith AN, York DF. (1997) Infant-maternal HIVspecific immunoglobulin Gl antibody ratios as an indicator of vertical transmission. AIDS 11: 11911193.

31. Kukolj G, Jones KS, Skalka AM. (1997) Subcellular localization of avian sarcoma virus and human immunodeficiency virus type 1 integrases. J. Virol. 71: 843-847.

32. Popov S, Rexach M, Zybarth G, et al. (1998) Viral protein $R(\mathrm{Vpr})$ regulates nuclear import of the HIV-1 preintegration complex. EMBO J. 17: 909917.

33. Stevenson $M$, Haggerty S, Lamonica CA, Meier CM, Welch SK, Wasiak AJ. (1990) Integration is not necessary for expression of human immunodeficiency virus type 1 protein products. $J$. Virol. 64: 2421-2425.

34. Zack JA, Arrigo SJ, Weitsman SR, Go AS, Haislip A, Chen IS. (1990) HIV-1 entry into quiescent primary lymphocytes: molecular analysis reveals a labile, latent viral structure. Cell 61: 213-222.

35. Li G, Simm M, Potash MJ, Volsky DJ. (1993) Human immunodeficiency virus type 1 DNA synthesis, integration, and efficient viral replication in growth-arrested T cells. J. Virol. 67: 3969-3977.

36. Haffar OK, Smithgall MD, Popov S, et al. (1998) CNI-H0294, a nuclear importation inhibitor of the human immunodeficiency virus type-1 genome, abrogates virus replication in infected activated 
peripheral blood mononuclear cells. Antimicrob. Agents Chemother. (In press)

37. Popov S, Dubrovsky L, Lee MA, et al. (1996) Critical role of reverse transcriptase in the inhibitory mechanism of CNI-H0294 on HIV-1 nuclear translocation. Proc. Natl. Acad. Sci. USA 93: 1185911864.

38. Gallay P, Stitt V, Mundy C, Oettinger M, Trono D. (1996) Role of the karyopherin pathway in human immunodeficiency virus type 1 nuclear import. J. Virol. 70: 1027-1032.

39. Gallay P, Hope T, Chin D, Trono D. (1997) HIV-1 infection of nondividing cells through the recognition of integrase by the importin/karyopherin pathway. Proc. Natl. Acad. Sci. USA 94: 9825-9830.

40. Schlossmann J, Neupert W. (1995) Assembly of the preprotein receptor MOM72/MAS70 into the protein import complex of the outer membrane of mitochondria. J. Biol. Chem. 270: 27116-27121.

41. Dworetzky SI, Lanford RE, Feldherr CM. (1988) The effects of variations in the number and sequence of targeting signals on nuclear uptake. J. Cell Biol. 107: 1279-1287.

42. Blomer U, Naldini L, Kafri T, Trono D, Verma IM, Gage FH. (1997) Highly efficient and sustained gene transfer in adult neurons with a lentivirus vector. J. Virol. 71: 6641-6649.

43. Vodicka MA, Koepp DM, Silver PA, Emerman M. (1998) HIV-1 Vpr interacts with the nuclear transport pathway to promote macrophage infection. Genes Dev. 12: 175-185.

44. Di Marzio P, Choe S, Ebright M, Knoblauch R, Landau NR. (1995) Mutational analysis of cell cycle arrest, nuclear localization and virion pack- aging of human immunodeficiency virus type 1 Vpr. J. Virol. 69: 7909-7916.

45. Zhao LJ, Mukherjee S, Narayan O. (1994) Biochemical mechanism of HIV-1 Vpr function. Specific interaction with a cellular protein. J. Biol. Chem. 269: 15577-15582.

46. Zhao LJ, Wang L, Mukherjee S, Narayan $O$. (1994) Biochemical mechanism of HIV-1 Vpr function. Oligomerization mediated by the $\mathrm{N}$-terminal domain. J. Biol. Chem. 269: 32131-32137.

47. Refaeli Y, Levy DN, Weiner DB. (1995) The glucocorticoid receptor type II complex is a target of the HIV-1 vpr gene product. Proc. Natl. Acad. Sci. USA 92: 3621-3625.

48. Mahalingam S, Ayyavoo V, Patel M, Kieber-Emmons T, Weiner DB. (1997) Nuclear import, virion incorporation, and cell cycle arrest/differentiation are mediated by distinct functional domains of human immunodeficiency virus type 1 Vpr. J. Virol. 71: 6339-6347.

49. Zufferey R, Nagy D, Mandel RJ, Naldini L, Trono D. (1997) Multiply attenuated lentiviral vector achieves efficient gene delivery in vivo. Nat. Biotechnol. 15: 871-875.

50. Hill CP, Worthylake D, Bancroft DP, Christensen AM, Sundquist WI. (1996) Crystal structures of the trimeric human immunodeficiency virus type 1 matrix protein: Implications for membrane association and assembly. Proc. Natl. Acad. Sci. USA 93: 3099-3104.

51. Popov S, Rexach M, Blobel G, Bukrinsky MI. (1998) Viral protein $R$ regulates docking of the HIV-1 preintegration complex to the nuclear pore complex. J. Biol. Chem. (In press) 